\title{
3D BODY SCAN AS ANTHROPOMETRIC TOOL FOR INDIVIDUALIZED PROSTHETIC SOCKS
}

\author{
Eva Lindell, Hanna Tingsvik, Li Guo, Joel Peterson* \\ The Swedish School of Textiles, University of Borås Allégatan 1, 50190 Borås, Sweden \\ ${ }^{*}$ Corresponding author. E-mail: joel.peterson@hb.se
}

\begin{abstract}
:
Every year, approximately 3,000 people in Sweden undergo amputation of a body part. The use of a prosthesis can greatly improve the quality of life for these people. To improve the fit and comfort of a prosthesis, a sock is used as an interface between the prosthesis socket and the stump. A three-dimensional (3D) body scanner can be used to take measurements that are used to produce individualized socks that improve fit and comfort. The standardized method for taking measurements with a $3 D$ body scanner often requires a standing position and hence a new scanning method is needed to improve the accessibility for $3 D$ body scanning. This study aimed to create a scanning scenario and an algorithm for scanning amputation stumps for individualizing prosthesis socks for upper-body amputations. Vitronic VITUSSMART LC 3D Body Scanner was used in this study. The results show a seated position with arms slightly away from the body, scanned at $45^{\circ}$ as the best. To measure the right upper arm and the left armpit, the best was to scan at a $315^{\circ}$ angle. Paired t-tests showed no significant differences compared with the $3 D$ body scanner of traditional manual measurements. The proposed method exhibited good relative reliability and potential to facilitate the customization of prosthetic socks for amputees.
\end{abstract}

\section{Keywords:}

3D Body Scanner, prosthetic sock, upper-body amputation, anthropometric measurement

\section{Introduction}

Every year, approximately 3,000 people in Sweden undergo one or more amputations, of which approximately $90 \%$ are of lower extremities and $10 \%$ are of upper extremities [1]. Vascular diseases most often necessitate amputation of lower extremities, whereas amputation of upper extremities is more common after a person undergoes a trauma [2]. An ill-fitting prosthesis can result in discomfort for the person who uses it, and the function of the prosthesis is reduced [3]. Textiles can provide a flexible and effective solution when using a prosthesis; one example is a prosthetic sock put on the stump of the amputee to reduce abrasion and discomfort [2]. The sock is the interface between the body and the prosthesis and can partially reduce discomfort and damage from abrasion and strain. Fit is important in clothing and fashion, and for prosthesis users, it is crucial. By personalizing the sock, the fit can be optimized. For this to be accomplished, accurate anthropometric data on the stump are needed.

Anthropometric data are of great importance in designing textile constructions for people and require measurement methods that are highly accurate and reliable. The traditional method of collecting anthropometric data is a manual measurement with measuring tape or calipers [4]. Standard ISO 7250 concerns anthropometric measurement, including precise instructions on which instruments should be used and how each measurement should be taken [5]. However, manual anthropometric measurement is time-consuming and has low repeatability, and the results often vary from one measurement occasion to the next and between different persons taking the same measurement $[4,6,7,8]$. Bragança et al. [9] stated some specific difficulties in performing manual measurement:

1. It is difficult for the test subject to hold a fixed position throughout the measurement session because it is timeconsuming.

2. Compression from the measuring tape gives measurements that may be too low.

3. Identification of anatomical landmarks on the body is subjective.

These limitations apply to a greater extent to anthropometric measurements taken by operators who lack experience.

One advantage of using a 3D body scanner to take measurements of the body is that it has high repeatability $[10,11]$. Another advantage of using 3D body scanners is that the scan images obtained can be re-evaluated without the person being measured needed to be present [12]. This makes the process of obtaining body measurements easier for people with various types of mobility disorders that make the static positioning 
required when taking manual measurements difficult. Dessery and Pallari [7] compared two different types of handheld 3D body scanners and manually taken measurements. They found that a 3D body scanner can provide more information, although the reliability of the measurements was found to depend on which scanner was used and the size of the scanned body part.

Anthropometric measurements form the basis of many standards and textile constructions. ISO Standard 20685-1:2018 aims to increase the comparability of body measurements measured with a 3D body scanner [13]. ISO Standard 20685-2:2018 aims to standardize the comparison of 3D body scanning data from different hardware and software systems [14]. These standards lack guidance on how people with one or more amputations should be scanned to obtain comparable and repeatable results, as shown by Sims et al. [4].

Previous attempts to scan people with amputations using 3D body scanners have shown that the software may not correctly register the data for the amputation stump. On the scanned image, a black shadow may appear that corresponds to unregistered data that the software did not detect. It has also been confirmed that 3D body scanners cannot register data in areas such as the crotch and axilla [15]. This makes it difficult to obtain measurements from the scanned image.

Furthermore, upper-arm width measurements by 3D body scanners have lower reliability than manual measurements [10]. A standing position presents several difficulties for a person with amputation of a lower extremity, both in getting onto the podium and maintaining balance during the scan, as it must be completely dark in the room. When measurements are taken either by traditional methods or by using 3D body scanners, it is necessary for the test subject to hold a specific position for a relatively long time, which presents difficulties for people with mobility impairments, such as people with amputations [4].

\subsection{Aim and research questions}

This study aimed to design a scanning scenario and an algorithm for 3D body scanning of an upper-limb (arm) amputation stump for individualizing the design of prosthetic stockings. The work was limited to amputation stumps of the upper limbs of the body and the use of 3D whole-body scanners. This study did not include comparisons between different types of 3D body scanners or different types of software. The test subjects were persons without mobility disorders.

Research questions addressed to this work were:

(RQ1) How can a scanning scenario for persons with amputations be designed?

(RQ2) How can a 3D body scanner be used to obtain information about the dimensions and shape of an amputation stump?

(RQ3) How can a scanning method be assessed with regard to validity and reliability?

\section{Experiments}

The experimental work was carried out in three main phases: pre-study, data collection, and data analysis. The equipment consisted of a digital measurement tool of a 3D body scanner with built-in software and a traditional measuring tape as a manual measurement tool. The values obtained from the scanner and the manual measurements were three length measurements $(L)$ and three circumference measurements $(C)$ for each arm (Figure 1). These were performed on both the left arm (L) and the right arm (R).

\subsection{Digital equipment}

The body scanner used was a full 3D body scanner from Human Solutions (Kaiserslautern, Germany), available at the University of Borås. The specification of the 3D body scanner is shown in Table 1. The scanner was calibrated every day as the tests were performed.

\section{$\underline{2.2 \text { Manual equipment }}$}

The manual measurements were performed with a Seca measuring tape (Table 2) and a Seca altimeter and scale.

Physical markers for direct placement on the body were produced with dimensions of approximately $1 \mathrm{~cm}$ in diameter and $0.5 \mathrm{~cm}$ in height, and they were attached directly to the skin with skin-friendly tape. These markers were used to ensure that the manual measurements were taken at the same locations as the digital measurements.

\section{$\underline{2.3 \text { Pre-study }}$}

A pre-study consisted of three phases (Figure 2) has been performed to identify an optimal scanning scenario. It was conducted to investigate the position held by the test subject and the angle this position was headed in the scanner that yielded an optimal image of the arms, primarily the upper

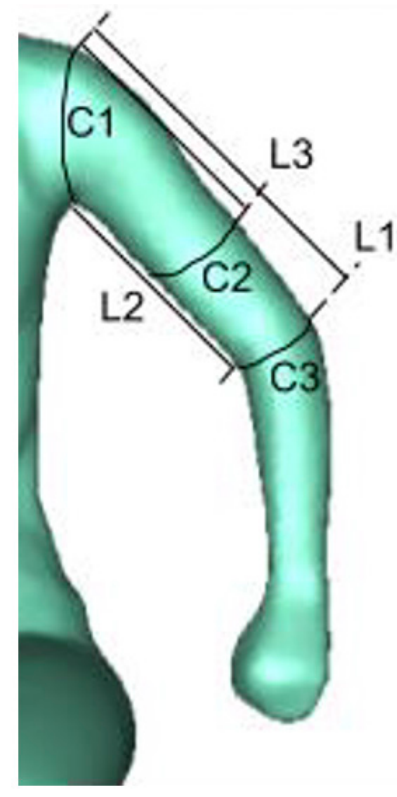

Figure 1. Upper-arm length and circumference measurement locations. 
Table 1. Specifications of the 3D body scanner used for the study

\begin{tabular}{|c|c|}
\hline Parameter & Value \\
\hline Software & Anthroscan 2014 3.0.7 \\
\hline Weight & Optic triangulation \\
\hline Measurement technique & 3 \\
\hline Number of sensor heads & Maximum error 3 mm \\
\hline Measurement accuracy & $12 \mathrm{~s}$ \\
\hline Scan time & $7 / \mathrm{cm}^{2}$ \\
\hline Point density & $2.98 \mathrm{~m}^{2}$ \\
\hline Scan capacity & \\
\hline
\end{tabular}

arms. The position and angle together constituted a scanning scenario that was developed in phase one, which, after the prestudy, was tested on eight voluntary test subjects in the data collection. In phase three of the pre-study, a 3D body scanner was programmed with a configuration file that created the new scanning scenario in the program.

The third phase of the pre-study was made in an attempt to deviate from the pre-programmed positions and measurements
Table 2. Specifications of the Seca measuring tape [16] used for manual measurement

\begin{tabular}{|c|c|}
\hline Parameter & Value \\
\hline Measurement range & $0-205 \mathrm{~cm}$ \\
\hline Division & $1 \mathrm{~mm}$ \\
\hline Diameter & $70 \mathrm{~mm}$ \\
\hline Height & $22 \mathrm{~mm}$ \\
\hline Weight of device & $50 \mathrm{~g}$ \\
\hline
\end{tabular}

within the 3D body scanner software. A new scanning scenario was developed that decided how to perform the physical positions. It was not possible to make the software perform the actual measurement automatically; this was performed with available functions in the program instead.

Phase one of the pre-study aimed to study how the scanner collects data about the body surface and how shadows in the image produced can be avoided in areas on the upper part of the body that often causes loss of data. To investigate this, four different positions were considered (Figure 3).

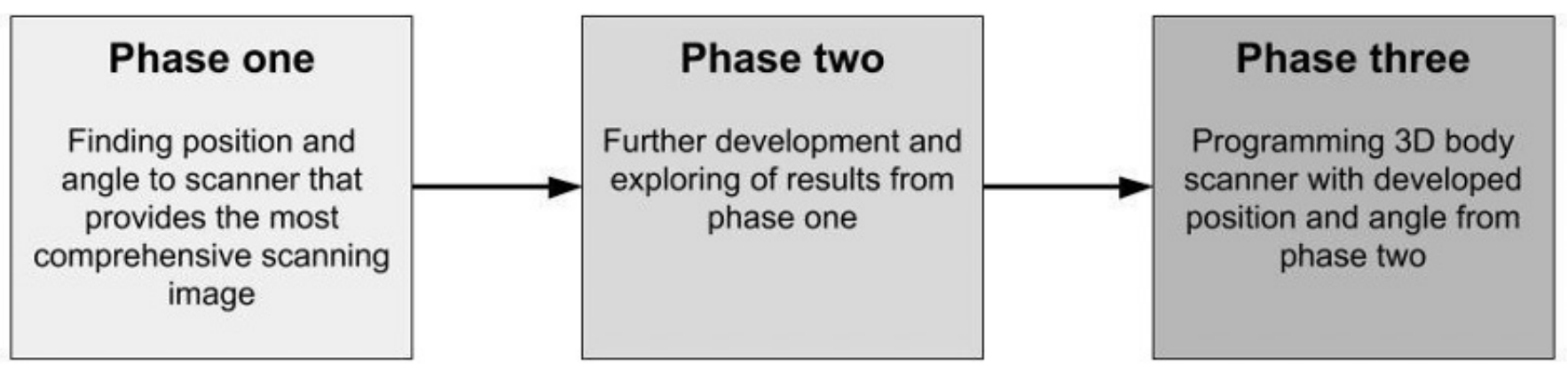

Figure 2. Overview of the pre-study, consisting of three phases to explore and develop the most comprehensive image from the 3D body scanner with the least amount of lost data.

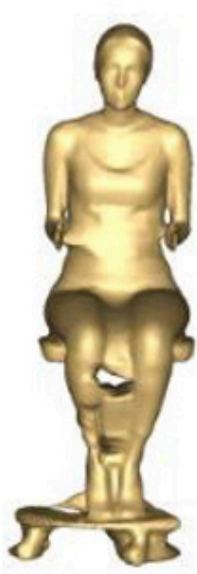

(a) Position D

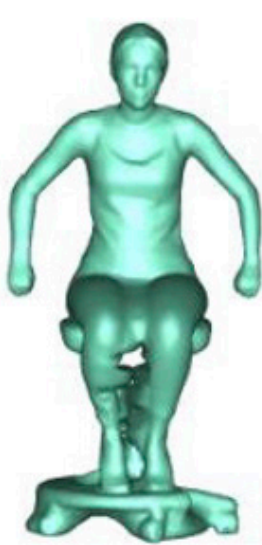

(b) Position X

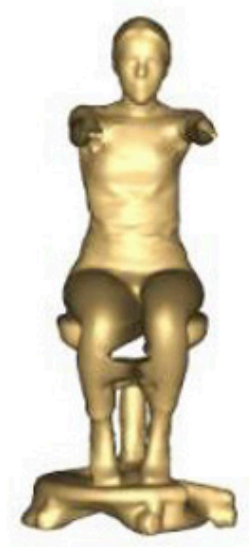

(c) Position Y

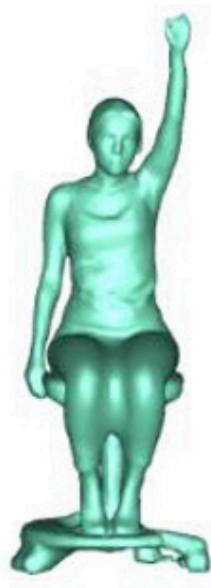

(d) Position Z

Figure 3. Positions examined in the pre-study. The positions were chosen in a way to optimize the reach of the 3D body scanner laser to the upper arms. 
Positions $D, X, Y$, and $Z$ were scanned at eight different angles relative to the reader of the scanner at intervals of $45^{\circ}$, as shown in Figure 4. This was done to investigate whether an optimal angle in relation to the three sensor heads in the 3D scanner could be identified that would generate an image with smaller shadows.

To evaluate the positions, the evaluation of hidden area protocol according to ISO 20685-2 [14] was used. The protocol was modified so that the areas evaluated were only on the upper body. The areas included in the evaluation were the shoulders, the outer lateral side of the upper arm, the armpit and torso under the armpit, the inside of the upper arm, the rear part of the upper arm, and the upper chest. The positions that produced the fewest and smallest shadows were then chosen to be further developed in phase two. The positions were tested with some modifications, varying the angle of the arms or positioning the arms farther behind in relation to the body.

Programming of the 3D body scanner software represents the third phase as shown in Figure 2. A new configuration file was written and programmed into the Anthroscan software. This programming produced a new scan scenario giving instructions concerning the different body positions for the test subject to hold and angles at which the scan was to be performed. Additional programming was carried out to program measurement scenarios into the software. This provided instructions in the program for placing the physical targets so that measurements were taken at the same points using the program as during the manual measurements. Attempts made to program the software to perform the measurements automatically for each scan were unsuccessful.

\subsection{Data collection}

The position and angle inside the scanning space that together constituted a scanning scenario were investigated in the pre-study, which in the second phase, data collection, was tested on eight voluntary test subjects. Tests for the selected measurements were performed both manually with the standardized measuring tape and with the help of the 3D body scanner software Anthroscan.

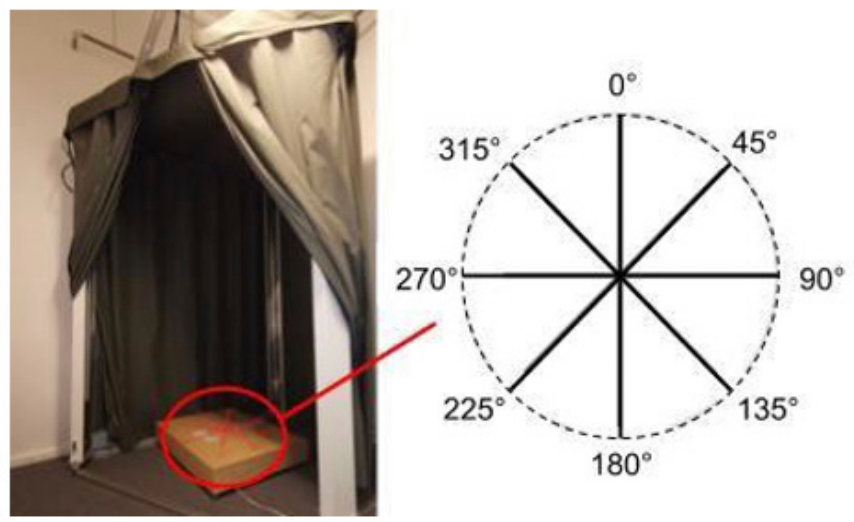

Figure 4. Scanning space and position angles used. The podium inside the 3D body scanner was fixed; hence, the exploration was done by having the test subject rotating their positions in relation center front of the scanning space.
Table 3. Information about study subjects (mean values followed by standard deviations in parentheses)

\begin{tabular}{|c|c|c|c|}
\hline & $\begin{array}{c}\text { Women } \\
(\mathbf{n}=\mathbf{4})\end{array}$ & Men $(\mathbf{n}=\mathbf{4})$ & Total $(\mathbf{n}=\mathbf{8})$ \\
\hline Age (year) & $26.0(2.94)$ & $25.5(1.29)$ & $25.8(2.1)$ \\
\hline Weight $(\mathrm{kg})$ & $59.33(5.40)$ & $80.4(13.38)$ & $69.9(14.7)$ \\
\hline Height $(\mathrm{cm})$ & $172.35(4.54)$ & $184.73(5.63)$ & $178.5(8.1)$ \\
\hline
\end{tabular}

Tests were conducted on eight adults, four men and four women, selected through personal contacts with the authors. Information about the test subjects is provided in Table 3.

Physical markers were placed on each test subject's body before manual measurement and scanning. Instructions were given on the seated position to be held during the scan and at what angle the position was to be oriented. The test subject was instructed to wear one tight-fitting thin garment that left the shoulder joint clearly visible and the arms unclothed. Before each scan, information about the test subject's age, gender, height, and weight was obtained. The body scan was performed two times at both angles (four scan images per test subject). The manual measurements were taken in the same position as in the scan and taken directly on the body, without pushing the tape into the skin. The total time for each scan and measurement session was in the range of 30-40 min. The manually taken measurements selected for validation were performed on both the left arm $(L)$ and the right arm (R).

\subsubsection{Manual measurements}

Manual measurements were performed directly on the body according to ISO 7250 [5] using the standardized tape measure. To ensure that both types of measurements were performed in the same way, the measurement technique was determined in advance. Figure 5 illustrates longitudinal and circumferential measurements with the tape measure.

\subsubsection{Digital measurement}

Measurements from 3D body scanner images were made using the software's length and cross-sectional measurement

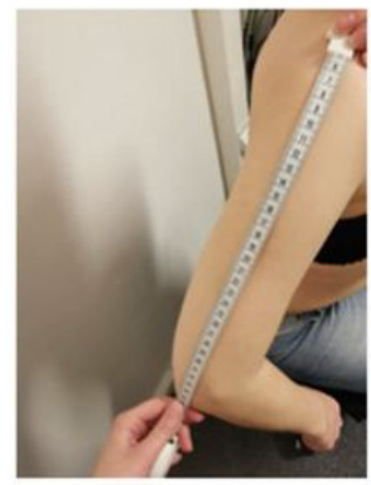

a) Length measurement

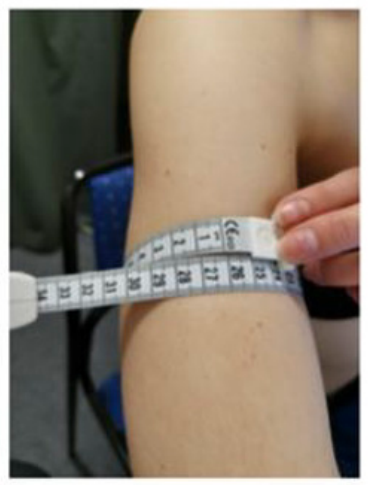

b) Circumference measurement
Figure 5. Manual measurement methods with Seca measuring tape. The instructions for how to perform this was taken from ISO 7250. 


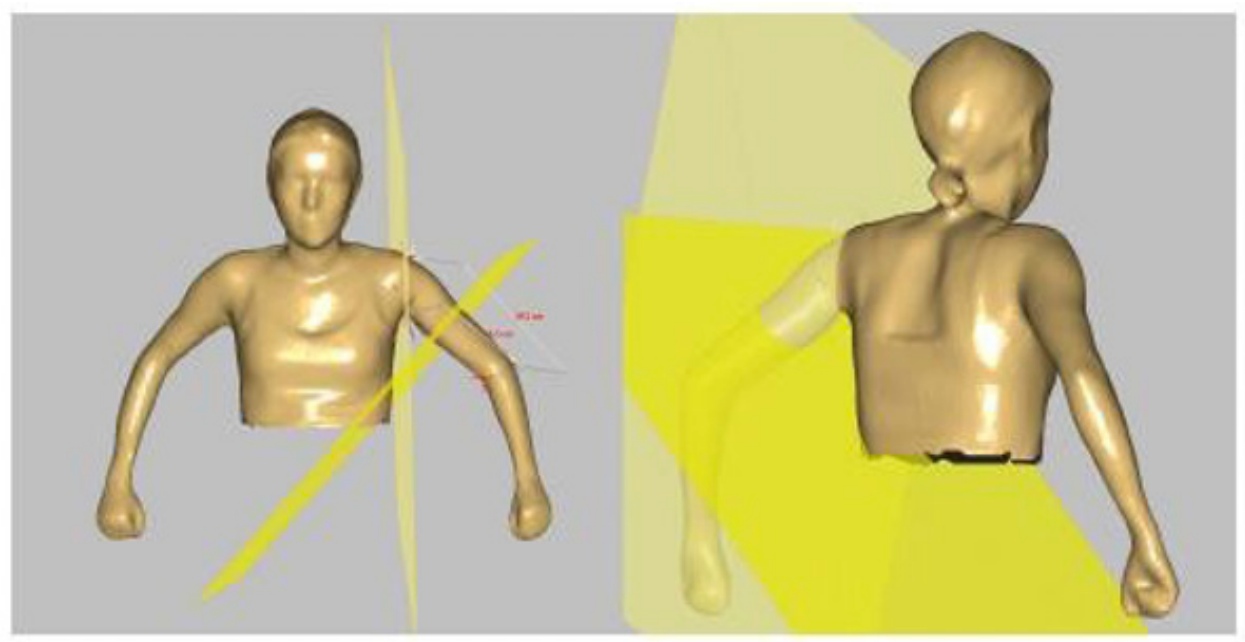

Figure 6. Use of cross-sections to obtain circumference measurements.

functions. Length measurements were taken between specified physical markers placed on the test subject's body before the measurements being taken. Circumference measurements were obtained using planar cross-sections at measurement locations (Figure 6).

\section{Results and discussion}

\subsection{Shapiro-Wilk's test and paired t-tests}

Shapiro-Wilks test results (not shown) indicated that the data were normally distributed. Paired $t$-tests (two sample $t$-tests with different variances) were conducted to compare the mean values for all twelve measurement locations (six on the left arm and six on the right arm). The results showed that there were no significant differences between the means of the manually and digitally obtained measurements (Table 4).

These results stand in contrast to those of another study [9] in which paired t-tests of manually obtained versus scanned measurements revealed significant differences in the mean values for shoulder width, waist circumference, seat circumference, lower circumference, knee circumference, and ankle circumference. The authors of that study attribute this to the difficulty of obtaining measurements of the body manually, which makes it more difficult to obtain the same measured values using both the manual and scanning methods. Another study [4] also detected significant differences between

Table 4. Paired $t$-test results for scanned and manually obtained measurements

\begin{tabular}{|c|c|c|c|}
\hline Measurement & $\boldsymbol{t}$ & $\mathbf{d f}$ & $\boldsymbol{p}$ (two-tailed test) \\
\hline LL1 & -0.4422 & 14 & 0.6651 \\
\hline LL2 & 0.3759 & 14 & 0.7127 \\
\hline LL3 & 0.0621 & 14 & 0.9514 \\
\hline LC1 & -0.5218 & 14 & 0.3050 \\
\hline LC2 & -0.3196 & 14 & 0.7540 \\
\hline LC3 & -0.6255 & 14 & 0.54170 \\
\hline
\end{tabular}

manually taken and scanned measurements, although the authors of that study commented that they should match and that the best agreement was achieved for measurement locations for which anatomical directives were easier to find. In [4], the observed average deviation (MAD) for arm length was $7 \mathrm{~mm}$, whereas, in the present study, MAD was $5 \mathrm{~mm}$ for the upper-arm length and $7 \mathrm{~mm}$ for the left arm's length. In [11], manual measurements were compared to those from a 3D full-body scanner, using a standardized dock. A t-test of the two types of measures showed that the 3D body scanner was significantly lower $(p<0.05)$ than manual measurements, but the $3 \mathrm{D}$ body scanner measurements had a smaller variance than measurements obtained using a handheld scanner.

\subsection{Results for the validity of measurement techniques}

The validity of the two measurement methods, manual and scanning, was assessed by calculating the MAD between the technical error of measurement (TEM) for each of the methods, manual and scanned.

\subsubsection{Average deviation}

The average deviation between the manually obtained values and the scanned values was calculated according to Eq. (1) and is shown for each measurement location as shown in Figure 7 .

$$
\mathrm{MAD}=\frac{1}{N} \sum_{i=1}^{n}\left(\mid \frac{1}{2} \sum_{j=1}^{r} x_{i j}-\sum_{j=1}^{r} y_{i j}\right)
$$

In this equation, $x$ represents the manual measurement, while $y$ represents the measurement taken by the 3D scanner. From Figure 7 we can see that the circumference measurements (c) exhibited greater differences than the length measurements $(\mathrm{L})$, which indicates a lower validity for these measures.

\subsubsection{Measurement error}

The values for TEM were calculated for both the manual and digital measurements using Eq. (2) and are reported in Table 5 in terms of length. 


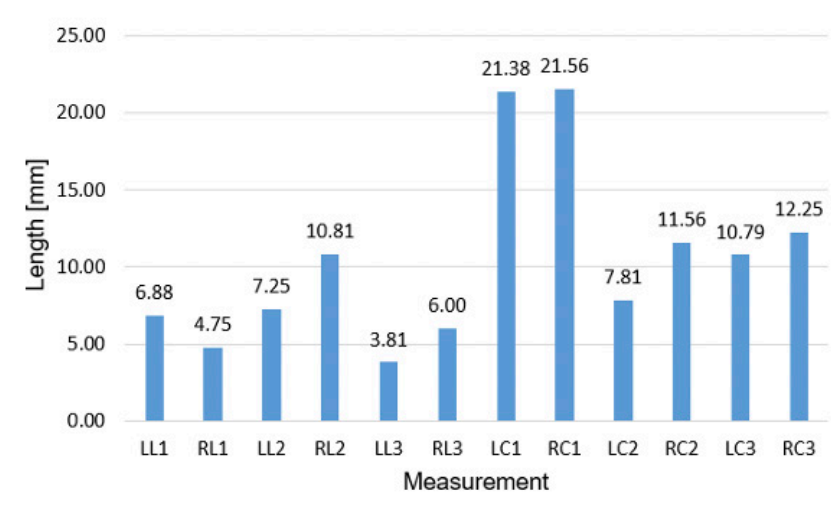

Figure 7. Results for mean average deviation (MAD).

$\mathrm{TEM}=\sqrt{\frac{\sum D^{2}}{2 N}}$

where $D$ is the difference between the manual and the scanned measurement and $N$ is the total number of measurements.

For 9 of the 12 measurements, the scanned values exhibited lower errors than the manually measured values. This means that the scanning measurement method has higher precision and a smaller measurement error than the manual measurement methods.

\subsection{Reliability}

The reliability of the two measurement methods was assessed by calculating the reliability constant $\left(R_{c}\right)$ and the coefficient of variance $(\mathrm{CV})$.

Table 5. Calculated measurement errors (TEM)

\begin{tabular}{|c|c|c|}
\hline Measurement & $\begin{array}{c}\text { Manual } \\
\text { measurements } \\
(\mathrm{mm})\end{array}$ & $\begin{array}{c}\text { Scanned } \\
\text { measurement } \\
(\mathrm{mm})\end{array}$ \\
\hline LL1 & 5.6 & $5.3^{*}$ \\
\hline LL2 & 6.8 & $4.9^{*}$ \\
\hline LL3 & 7.6 & $5.3^{*}$ \\
\hline LC1 & 24.6 & $8.0^{*}$ \\
\hline LC2 & 3.3 & $6.0^{*}$ \\
\hline LC3 & 6.5 & 8.8 \\
\hline RL1 & 5.4 & $4.4^{*}$ \\
\hline RL2 & 8.6 & $4.6^{*}$ \\
\hline RL3 & 7.6 & $4.8^{*}$ \\
\hline $\mathrm{RC} 1$ & 10.3 & 10.5 \\
\hline $\mathrm{RC} 2$ & 2.9 & 8.5 \\
\hline RC3 & 5.6 & $4.9 *$ \\
\hline
\end{tabular}

\begin{tabular}{|c|c|c|}
\hline Measurement & Manual (no units) & Scanned (no units) \\
\hline LL1 & 0.92 & $0.94^{*}$ \\
\hline LL2 & 0.94 & $0.97^{*}$ \\
\hline LL3 & 0.87 & $0.95^{*}$ \\
\hline LC1 & 0.83 & $0.98^{*}$ \\
\hline LC2 & 0.99 & 0.96 \\
\hline LC3 & 0.96 & 0.91 \\
\hline RL1 & 0.92 & $0.96^{*}$ \\
\hline RL2 & 0.86 & $0.97^{*}$ \\
\hline RL3 & 0.89 & $0.95^{*}$ \\
\hline RC1 & 0.95 & $0.97^{*}$ \\
\hline RC2 & 0.99 & 0.93 \\
\hline RC3 & 0.95 & $0.97^{*}$ \\
\hline
\end{tabular}

where TEM is the technical error of measurement (Eq. 2) and $\mathrm{SD}$ is the standard deviation. $R_{c}$ describes the relationship between the measurement accuracy and the standard deviation. If the expected measurement error is larger than the standard deviation, the measurement has low reliability.

The values of the reliability constant calculated in this study (Table 6) also show that the 3D body scanner has better reliability and lower variance than the manual measurement method. One factor that may have affected the reliability values achieved in this study include the program's software integration not being done automatically but rather having to be performed individually for each scanned image using the program's measuring features.

\subsubsection{Coefficient of variation}

The internal reliability is expressed by the CV calculated as shown in Eq. (4). The calculated values are shown in Table 7.

$\mathrm{CV}=\frac{\mathrm{SD}}{\tilde{x}}$

Table 6. Calculated reliability constant $R_{c}$ 
Table 7. Calculated coefficients of variation

\begin{tabular}{|c|c|c|}
\hline Measurement & Manual (no units) & Scanned (no units) \\
\hline LL1 & 0.06 & 0.06 \\
\hline LL2 & 0.11 & 0.12 \\
\hline LL3 & 0.14 & 0.15 \\
\hline LC1 & 0.16 & 0.14 \\
\hline LC2 & 0.12 & 0.11 \\
\hline LC3 & 0.12 & 0.11 \\
\hline RL1 & 0.05 & 0.07 \\
\hline RL2 & 0.09 & 0.11 \\
\hline RL3 & 0.14 & 0.15 \\
\hline RC1 & 0.12 & 0.14 \\
\hline RC2 & 0.12 & 0.11 \\
\hline RC3 & 0.10 & 0.10 \\
\hline
\end{tabular}

The measurement points with $\mathrm{CV}$ below 0.1 for both measurement methods were LL1 and RL1. The manual measurement method also had a CV below 0.1 for location RL1. All of these were length measurements.

\subsection{Answers to the research questions}

(RQ1) How can a scanning scenario be designed for people with an amputation?

For people with an amputation, maintaining balance was perceived to be difficult during the scanning; therefore, a sitting position with the arms away from the body was developed. Even though persons with upper-limb amputations may not have difficulties keeping a standing position, developing a seated position extends the use and availability to persons with, for example, multiple amputations or other mobility disorders. The subject was scanned at a $45^{\circ}$ angle to the front in the body scanner to obtain measurements on the left upper arm and right axilla and at a $315^{\circ}$ angle to obtain measurements on the right upper arm and left axilla.

(RQ2) How can a 3D body scanner be used to obtain information on the dimensions and shape of an amputation stump?

A 3D body scanner can provide anthropometric data for the arms with good reliability but lower validity than manual measurements. Further studies on people with amputated upper extremities are needed.
(RQ3) How can such a scanning method be assessed with regard to validity and reliability?

The validity of the study can be assessed in terms of the average deviation and compared using ISO 20685-1. The external reliability is assessed using the reliability constant (R), and the internal coefficient is evaluated with the CV. The result of the study shows good external reliability but lower internal reliability for the scanning method. To strengthen the study's statistical results, a sample set of at least 85 persons should be employed.

The results of the pre-study show good scanning images without shading in the eligible areas. When the same position was used for the test subjects and scanned, the results were different. Large portions of shadows were shown on the lower part of the arms on some of the scans of the test subjects, while others yielded good pictures without shadows. The scan images with shadows had reasonably good results for the length of the arm. The current circumference could be determined, but because of the shadows, data were lost. This variation probably depends on how well the position is maintained during the scan, some variation between test subjects is to be expected. This study focused on body scanning of amputation stump for individual fitting of prosthetic socks for the research project Scan-To-Knit. For the finished scan image to be used to create an individualized prosthetic sock, the image needs to be converted to a computer program for use with a flat knitting machine. Further studies should be done on this transformation to facilitate the implementation of the scanning scenario in the research project.

\section{Conclusions}

This study aimed to design a scanning scenario and an algorithm for 3D body scanning of upper-limb amputation stubs for individualizing prosthetic stockings. The results show that it is possible to use 3D whole-body scanners to scan individual parts of the body without using the software's built-in automated measurement system. Further development and research are needed to improve the method. It was not possible in this study to program the measurement steps for the 3D body scanner software; the existing measuring tools available in the software system were used. Programming the software to take new measurements automatically would probably improve the method's measurement accuracy.

\section{Acknowledgments}

The Knowledge Foundation has funded the research project Scan to Knit-A Smart Textile Platform for Personalized Prosthetic Textiles at the Swedish School of Textiles, University of Borås, Sweden. The authors would like to express their appreciation to the industrial partners in the research project, especially the Lindhe Xtend $A B$ company. 


\section{References}

[1] Sandsjö, L., Guo, L. (2018). Scan-to-knit-A platform for personalised smart textiles research and development with a special focus on prosthetics. In ISEK 2018-The XXII Congress of the International Society of Electrophysiology and Kinesiology, 222-223.

[2] Carroll, K., Edelstein, J. (2006). Prosthetics and patient management: A comprehensive clinical approach (1st ed.). Slack, Thorofare, NJ.

[3] Geil, M. D. (2005). Consistency and accuracy of measurement of lower-limb amputee anthropometrics. The Journal of Rehabilitation and Development 42(2), 131-140. doi: 10.1682/JRRD.2004.05.0054.

[4] Sims, R. E., Marshall, R., Gyi, D. E., Summerskill, S. J., Case, K. (2012). Collection of anthropometry from older and physically impaired persons: Traditional methods versus TC $23 D$ body scanner. International Journal of Industrial Ergonomics, 42(1), 65-72. doi: 10.1016/j. ergon.2011.10.002.

[5] SS-ENISO 7250-1:2017. Basichuman bodymeasurements for technological design-Part 1: Body measurement definitions and landmarks.

[6] Troynikov, O., Ashayeri, E. (2011). 3D body scanning method for close-fitting garments in sport and medical applications. HFESA 47th Annual Conference 2011, 1116, doi: 10.15221/11.239.

[7] Dessery, Y., Pallari, J. (2018). Measurements agreement between low-cost and high-level handheld 3D scanners to scan the knee for designing a $3 D$ printed knee brace. PLoS One, 13(1), e0190585. doi: 10.1371/journal.pone.0190585.

[8] Seminati, E., Canepa Talamas, D., Youong, M., Twiste, M., Dhokia, V., et al. (2017). Validity and reliability of a novel $3 D$ scanner for assessment of the shape and volume of amputees' residual limb models. PLoS One 12(9), e0184498. doi: 10.1371/journal.pone.0184498.
[9] Bragança, S., Arezes, P., Carvalho, M., Ashdown, S. P., $X u$, B., Castellucci, I., et al. (2017). Validation study of a Kinect based body imaging system. Work, 57(1), 9-21.

[10] Kuehnapfel, A., Ahnert, P., Loeffler, M., Broda, A., Scholz, M. (2016). Reliability of $3 D$ laser-based anthropometry and comparison with classical anthropometry. Scientific Reports (Nature Publisher Group). 6(5), 1-11. doi: 10.1038/srep26672.

[11] Xia, S., Guo, S., Li, J., Istook, C. (2018). Comparison of different body measurement techniques: $3 D$ stationary scanner, 3D handheld scanner, and tape measurement. The Journal of the Textile Institute. 110(6), 1-11. doi: 10.1080/00405000.2018.1541437.

[12] Tomkinson, G. R., Shaw, L. G. (2013). Quantification of the postural and technical errors in asymptomatic adults using direct 3D whole body scan measurements of standing posture. Gait \& Posture, 37(2), 172-177. doi: 10.1016/j. gaitpost.2012.06.031.

[13] SS-EN ISO 20685-1:2018. Ergonomics-3D scanning methodologies for internationally compatible anthropometric databases - Part 1: Evaluation protocol for body dimensions extracted from 3D body scans.

[14] SS-EN ISO 20685-2:2018. Ergonomics-3D scanning methodologies for internationally compatible anthropometric databases - Part 2: Evaluation Protocol of surface shape and repeatability of relative landmark positions.

[15] Elbrecht, P., Palm, K. J. (2014). Precision of 3D body scanners. IEEE 18th International Conference on Intelligent Engineering Systems INES 2014, Tihany, 2014, 127-132. doi: 10.1109/INES.2014.6909355.

[16] Seca (2019). Seca 201 Ergonomic circumference measuring tape. Retrieved May 20, 2019. Website: https:// www.seca.com/fileadmin/documents/product_sheet/ seca_pst_201_en_int.pdf. 